\title{
Enzymatic modification of corn starch influences human fecal fermentation profiles
}

Angela Dura ${ }^{\dagger \dagger}$, Devin J Rose ${ }^{\dagger}$, Cristina M Rosell* ${ }^{\star}$

"Institute of Agrochemistry and Food Technology (IATA-CSIC), Avenida Agustin Escardino, 7, Paterna 46980, Valencia, Spain. E-mail: andudemi@iata.csic.es; crosell@iata.csic.es Phone number +34 963900022. Fax number: +34 963636301

${ }^{\dagger}$ Department of Food Science \& Technology, University of Nebraska-Lincoln, Lincoln, NE, USA. 268 Food Innovation Center Lincoln, NE 68588-6205. E-mail: drose3@unl.edu Phone number: +1 4024722831

*Corresponding author: Cristina M. Rosell. Phone number +34 963900022. Fax number: +34 963636301; E-mail: crosell@iata.csic.es 


\section{ABSTRACT}

2 Enzymatically modified starches have been widely used in food applications to develop new

3 products but information regarding digestion and fecal fermentation of these products is sparse.

4 The objective of this study was to determine the fermentation properties of corn starch modified

5 with $\alpha$-amylase, amyloglucosidase or cyclodextrin glycosyltransferase and the possible role of

6 hydrolysis products. Samples differed in their digestibility and availability to be fermented by the

7 microbiota, resulting in differences in microbial metabolites produced during in vitro

8 fermentation. The presence or absence of hydrolysis products and gelatinization affected starch

9 composition and subsequent metabolite production by the microbiota. Amyloglucosidase-treated

10 starch led to the greatest production of short and branched chain fatty acid production by the

11 microbiota. Results from this study could be taken into consideration to confirm the possible nutritional claims and potential health benefits of these starches as raw ingredients for food development.

KEYWORDS: Corn starch, enzymatic modification, short chain fatty acids, digestion, fermentation 


\section{INTRODUCTION}

Enzymes play important roles in many industrial applications, and can be considered processing aids to improve food quality attributes. Enzymes are widely use in the cereal food industry (reviewed in Rosell and Dura). ${ }^{1}$ In these products, starch modification by enzymes provides food ingredients that could not be produced by chemical or physical methods. ${ }^{2}$ Various enzyme modifications have been attempted to modify intrinsic properties of starch, increase water holding capacity or heat resistant behavior, minimize syneresis, improve thickening, and to achieve overall desired functionality of end products. ${ }^{3}$ Hydrolases like $\alpha$-amylases (AM) and amyloglucosidases (AMG) have been extensively used as replacements of acid hydrolysis in the production of starch hydrolysates. ${ }^{4}$ Another attractive opportunity is the production of porous starches by enzymatic treatments to extend their absorption capacity. Initially, porous starch was obtained by the joint action of AM and AMG on corn starch. ${ }^{2}$ These changes in the starch structure by the individual action of AM or AMG were also observed by Dura et al., ${ }^{5}$ increasing the susceptibility of the starch to be digested and modifying hydration properties and pasting parameters. Depending on the type and level of hydrolytic enzyme, it is possible to modulate the number and size of the pores on the surface of the granule, and therefore their adsorption capacity. ${ }^{6}$ Further studies have shown that enzymatic modification with cyclodextrin glycosyltransferase (CGTase) modifies pasting behavior in a $\mathrm{pH}$ dependent manner. ${ }^{7}$ Starch modification by CGTase seemed to hydrolyze the amorphous part of the starch granule, which led to small pores on the surface, with a simultaneous release of oligosaccharides and production of cyclodextrins. Other studies have reported that enzymatic modification of starches alters chain length, branch density and crystalline structure. ${ }^{8-9}$ Those studies confirmed the morphological 
changes of the porous starches, but no information exists about the impact of those changes on their digestion performance.

Diverse studies have pointed out the relevance of structural changes in food digestibility. For instance, in vitro starch digestion that simulates stomach and small intestinal conditions revealed that the increase in starch branch density and the crystalline structure in treated starches contributed to slow digestion. ${ }^{10}$ An increase in resistant starch was obtained when altering the crystallinity of the starch by treatment with $\alpha$-amylase and pullulanase. ${ }^{11}$ In addition, in vitro fecal fermentation coupled to in vitro digestion can provide information about how dietary components, such as resistant starch, are metabolized by gut bacteria. In vitro fermentation tests provide an insightful opportunity to study the function of the microbiota, i.e., to determine how the microbiota utilize certain substrates, which is difficult to do in vivo in humans because of the complexity of diets. ${ }^{12}$ Furthermore, in vitro fermentations allow for quantitative measurement of metabolites produced by the microbiota upon fermentation of specific substrates, ${ }^{13-14}$ which have importance in the health of the host. ${ }^{15-18}$ The most important end products of fermentation are short chain fatty acid (SCFA), principally acetate, propionate, and butyrate, and branch chain fatty acid (BCFA) that are then absorbed and metabolized by the host. SCFA are produced mainly from carbohydrate fermentation and have health benefits, whereas BCFA are a result of proteolytic fermentation and may have deleterious effects. ${ }^{19}$ In vitro fermentation and SCFA production have been useful to estimate the impact of dietary carbohydrates on human health. ${ }^{20-}$ ${ }^{23}$ However, while several studies have reported on the fermentation properties of resistant starches, no studies have reported on the susceptibility of structurally modified starches to be fermented by gut bacteria. ${ }^{24}$ The objective of this research was to determine the impact of structural modification of starch granules on the in vitro digestion and fermentation. For that 
purpose different enzymatically modified starches have been used to get a deeper understanding on how diverse porous corn starches and microbes interact with gastrointestinal microbiota and the types of metabolites that are produced upon fermentation.

\section{MATERIALS AND METHODS}

\section{Materials and reagents}

Corn starch samples were purchased from Daesang Corporation (Korea). Fungal $\alpha$-amylase (AM, EC 3.2.1.1) (Fungamyl® 2500SG, declared activity $2500 \mathrm{FAU} / \mathrm{g}$ product), amyloglucosidase (AMG, EC 3.2.1.3) (amyloglucosidase $1100 \mathrm{~L}$, declared activity $1100 \mathrm{AGU} / \mathrm{g}$ product) and cyclodextrin glycosyltransferase (CGTase, EC 2.4.1.19) from Thermoanaerobacter sp (Toruzyme ${ }^{\circledR} 3.0 \mathrm{~L}$, declared activity $3 \mathrm{KNU} / \mathrm{mL}$ product) were kindly provided by Novozyme (Bagsværd, Denmark). Chemical reagents from Sigma-Aldrich (Madrid, Spain) were of analytical grade.

\section{Methods}

\section{Samples preparation}

Corn starch $(10.0 \mathrm{~g})$ was suspended in $50 \mathrm{~mL}$ of $20 \mathrm{mM}$ sodium acetate buffer at $\mathrm{pH} 4.0$ or in sodium phosphate buffer at $\mathrm{pH}$ 6.0, for enzymatic modification with AMG or AM and CGTase, respectively. ${ }^{5,7}$ Enzymes (4 U of AMG/g starch, $5 \mathrm{U}$ of AM/g starch or $0.32 \mathrm{U}$ of CGTase/g starch) were then added to the starch suspension and suspensions were kept in a shaking water bath $(50 \mathrm{rpm})$ at $50{ }^{\circ} \mathrm{C}$ for $48 \mathrm{~h}$. Water $(50 \mathrm{~mL})$ was then added to the suspensions and the mixture was homogenized with a Polytron Ultraturrax homogenizer IKA-T18 (IKA Works, Inc. Wilmington, DE, USA) for $1 \mathrm{~min}$ at 14,000 rpm. Samples were centrifuged for $15 \mathrm{~min}$ at $7,000 \times g$ and $4{ }^{\circ} \mathrm{C}$. The starch pellets were washed twice with $50 \mathrm{~mL}$ of water and centrifuged 
again using the same conditions. Supernatants were pooled and placed in a boiling water bath for 10 min to inactivate enzymes.

For each enzyme, two enzymatically treated corn starches were prepared, the washed enzymatically treated corn starch (AMG-W; AM-W; CGT-W) and the other one containing the hydrolysis products (AMG-NW; AM-NW; CGT-NW) that were added back to assess the role of the water soluble hydrolysis products. Samples were freeze-dried and kept at $4{ }^{\circ} \mathrm{C}$ for further analyses.

Samples were also analyzed in the gelatinized $(\mathrm{G})$ and non-gelatinized (NG) forms. To obtain gelatinized samples, treated and untreated corn starch samples $(10 \mathrm{~g})$ were suspended in $40 \mathrm{~mL}$ of distilled water and incubated $15 \mathrm{~min}$ at $100{ }^{\circ} \mathrm{C}$. Samples were then freeze-dried and kept at 4 ${ }^{\circ} \mathrm{C}$ for further analyses. Native corn starch (N) was used as the control sample. Two batches were prepared for each sample.

\section{In vitro digestion}

The digestion process consisted of a simulated gastric digestion followed by a small intestinal phase (Figure 1), according to the method described by Yang et al. ${ }^{25}$ Briefly, samples (25 g) were suspended in $300 \mathrm{~mL}$ distilled water. Eight $\mathrm{mL}$ of $1 \mathrm{M} \mathrm{HCl}$ was added to the sample to reduce the $\mathrm{pH}$ to 2.5 . Ten milliliters of $100 \mathrm{mg} / \mathrm{mL}$ freshly prepared pepsin (P-700; Sigma, St. Louis, MO. USA) dissolved in $50 \mathrm{mM} \mathrm{HCl}$ was added and the mixture was placed on an orbital shaker at $250 \mathrm{rpm}$ (MaxQ 7000, $150 \mathrm{rpm}$; Barnstead, Dubuque, IA, USA) at $37^{\circ} \mathrm{C}$ for $30 \mathrm{~min}$ to achieve the gastric phase. The small intestinal phase was initiated with the addition of $50 \mathrm{~mL}$ of $0.1 \mathrm{M}$ sodium maleate buffer $\left(\mathrm{pH}=6\right.$, containing $1 \mathrm{mM} \mathrm{CaCl}_{2}$ ) and $20 \mathrm{~mL}$ of $1 \mathrm{M} \mathrm{NaHCO}_{3}$ to bring the $\mathrm{pH}$ to 6.9 . Fifty milliliters of $125 \mathrm{mg} / \mathrm{mL}$ pancreatin (P-7545, Sigma) dissolved in the sodium maleate buffer and $6500 \mathrm{U}$ of amyloglucosidase $(0.56 \mathrm{~g}$ of $11600 \mathrm{U} / \mathrm{g}$; A7255, Sigma) 
were then added and samples were incubated in a shaking water hath at $37^{\circ} \mathrm{C}$ for $6 \mathrm{~h}$. Digested contents were then poured into dialysis tubing (molecular weight cutoff $12,000-14,000$ ) and dialyzed for three days against distilled water with changing of the water every $2-3 \mathrm{~h}$. The retentate was then frozen $\left(-20^{\circ} \mathrm{C}\right)$ overnight and then freeze dried. Total starch content was determined before and after the in vitro digestion following AACC approved method 76-13.01 16 using a kit (K-TSTA, Megazyme, Bray, Ireland). Digestion assays were run in triplicate.

\section{In vitro fermentation}

In vitro fecal fermentation was performed according to the method described by Arcila et al. ${ }^{26}$ with minor modifications (Figure 1). In short, $15 \mathrm{mg}$ of digested, freeze-dried material was suspended in $1 \mathrm{~mL}$ of sterile fermentation medium consisting of (per liter) peptone ( $2 \mathrm{~g}$, BP1420100; Fisher Scientific, Pittsburgh, PA, USA), yeast extract (2 g; CAS8013-01-2; Alfa Aesar, Ward Hill, MA, USA), bile salts (0.5 g; S71919-1; Fisher Science; Hannover Park, IL, USA), $\mathrm{NaHCO}_{3}(2 \mathrm{~g}), \mathrm{NaCl}(0.1 \mathrm{~g}), \mathrm{K}_{2} \mathrm{HPO}_{4}(0.08 \mathrm{~g}), \mathrm{MgSO}_{4} \cdot 7 \mathrm{H}_{2} \mathrm{O}(0.01 \mathrm{~g}), \mathrm{CaCl}_{2} \cdot 6 \mathrm{H}_{2} \mathrm{O}(0.01 \mathrm{~g}), \mathrm{L}-$ cysteine hydrochloride (0.5 g; CAS52-89-1; Sigma), hemin (50 mg; CAS16009-13-5; Sigma), Tween 80 (2 mL; P8074), vitamin K (10 $\mu \mathrm{L}$; 84-80-0; Sigma), and 0.025\% (w/v) resazurin solution (4 mL; CAS62758-13-8; Sigma), hydrated and placed it in the anaerobic hood overnight.

Fresh fecal samples were collected from three healthy individuals with no record of gastrointestinal abnormalities or antibiotic administration in the last 6 months. The fecal slurry was prepared by blending each fecal sample separately with sterile phosphate buffered saline in the ratio of 1:9 (w/v) using a hand blender for $1 \mathrm{~min}$ and then were filtered through four layers of cheesecloth. Tubes were then inoculated with $0.1 \mathrm{~mL}$ of fecal slurry, capped, placed at a $45^{\circ}$ angle, and incubated at $37^{\circ} \mathrm{C}$ with shaking (MaxQ 7000, $140 \mathrm{rpm}$ ). Samples were taken at 
specific time points $(0,4,8,12$ and $24 \mathrm{~h})$ stopping the reaction with $0.4 \mathrm{~mL}$ of $\mathrm{NaOH} 7.5 \mathrm{M}$ mixed with SCFA standard (7 mM 2-ethylbutyric acid). Samples were then transferred to a freezer $\left(-80^{\circ} \mathrm{C}\right)$ until analysis. Total starch content was analyzed after the in vitro fecal fermentation as described above. Three replicates were carried out to obtain the average.

\section{Short chain fatty acid determination}

Samples for SCFA were quantified by gas chromatography (Clarus 580, PerkinElmer, MA, USA) equipped with a capillary column (Elite-FFAP, $15 \mathrm{~m} \times 0.25 \mathrm{mminner}$ diameter $\times 0.25 \mu \mathrm{m}$ film thickness, PerkinElmer) and detected with a flame ionization detector, according to Arcila et al. ${ }^{26}$ SCFA were quantified by calculating response factors for each SCFA relative to 2ethylbutyric acid using injections of pure standards.

\section{Data analysis}

All treatments were performed in triplicate. Compositional data were analyzed using a threefactor (enzyme, washed/unwashed, gelatinization) ANOVA, including the two-way and threeway interactions, using Statgraphics Centurion XV software (Bitstream, Cambridge, N). When ANOVA indicated significant $F$ values $(P<0.05)$, multiple sample comparisons were also performed by Fisher's least significant differences (LSD) test to differentiate means with 95\% confidence. The correlation matrix was also calculated using Statgraphics Centurion XV software. All data were reported on a wet weight basis.

For SCFA analysis, the repeated measure of time was also included in the ANOVA model. For detecting significant effects a four factor ANOVA with interaction was performed. Significant differences among samples were determined using Bonferroni’s least significant difference (LSD) test with $\alpha=0.05$. Data were analyzed using SAS software (version 9.2, SAS Institute, Cary, NC USA). 


\section{RESULTS AND DISCUSSION}

\section{In vitro digestion of enzymatically treated starches}

Modified starches were in vitro digested and the impact of gelatinization was also assessed. The starch content was determined before and after the in vitro digestion (Figure 2). Significant differences $(P<0.05)$ were found among samples regarding digested starch for non-gelatinized samples (Figure 2A) and gelatinized samples (Figure 2B). When comparing treated starches in granular form, it was observed that the enzymatic modification influenced the action of digestive enzymes. The CGTase-NW sample showed the highest digestibility among all samples (Figure 2A). The presence of hydrolysis products influenced the quantity of starch digested. Although no significant differences were found, lower amount of starch was digested in the samples containing hydrolysis products (AM-NW, AMG-NW), which was expected due to product inhibition. This trend was not observed in the case of CGTase-NW starch, likely due to the low amount of oligosaccharides released during starch treatment ${ }^{6}$, which induced higher digestion of the starch granules. The digestion of this CGTase-NW starch was even higher than the native starch, which could be attributed to the presence of very small surface pores that increase the starch susceptibility to be digested. ${ }^{24}$ Among washed samples, digestibility of AMG-W starch was higher than CGTase-W, which was ascribed to the large surface holes that are present in this type of porous starch. ${ }^{5}$ AMG, AM and CGTase are all hydrolytic enzymes that affect the amorphous and crystalline domains of starch and increased the exposure of the granule to subsequent digestion. ${ }^{5,7}$

Differences in the digestion of the starches were intensified after gelatinization (Figure 2B). CGTase-NW sample gave the highest percentage of digestible starch among all samples. The gelatinization process encompassed the rearrangement of the starch granules and loss of 
crystallinity, transforming the starch granule into a more amorphous structure and favoring enzymes access to the starch chains. ${ }^{27}$ Therefore, gelatinization even augmented the susceptibility of CGTase-NW to be digested. Again, non-washed samples (AM-NW, AMG-NW) containing the hydrolysis products displayed lower digestion than their washed counterparts. AMG treated samples underwent higher digestion than those treated with AM. Previous studies showed that AMG action on the starch granules was greater than that induced by AM, ${ }^{5}$ affecting the crystalline structure, which in consequence favored the chain disentanglement during gelatinization and its further hydrolysis by digestive enzymes.

\section{In vitro fecal fermentation and SCFA production}

Microbial metabolites produced during fecal fermentation of the modified corn starch samples was affected by several of the treatment variables and their interactions (Table 1). The enzymatic treatment and the granule state (gelatinized or non-gelatinized) affected the production of BCFA and there was also an interaction between those factors. Only the enzymatic treatment provoked a significant effect on the production of propionate. The amount of starch remaining after fecal fermentation was significantly affected by the interaction among enzymatic treatment, hydrolysis products, and gelatinization. In addition, significant effects were observed at specific times of the fermentation process. Total SCFA, acetate and propionate exhibited a rapid increase during the first eight hours of fermentation, and thereafter slowed (Figure 3). Total SCFA were found to be significantly different at the end of the fermentation, showing lower values for those samples treated with AM or CGTase compared with the untreated sample and that treated with AMG. Propionate production showed significant differences from $12 \mathrm{~h}$ of fermentation: AMG treated sample resulted in higher propionate production compared with the other samples. Likewise, the 
AMG-treated sample resulted in significantly more butyrate production compared with the other samples.

SCFA production, especially butyrate production, is dependent on the presence of fermentable carbohydrate substrate. The higher SCFA production in the AMG-treated sample suggested that AMG treatment of starch resulted in more microbial available carbohydrates during fermentation. Given that Western diets are generally lacking in microbial available carbohydrates, ${ }^{28}$ AMG-treated starches may provide an important source of energy for the microbiota. An adequate carbohydrate supply promotes the growth of beneficial, saccharolytic members of the microbiota, such as Roseburia, Eubacterium rectale and Ruminococcus bromii that otherwise are outcompeted by more resilient but less beneficial genera such as Alistipes, Bilophila and Bacteroides. ${ }^{29}$ Roseburia and Eubacterium rectale especially are important butyrate producers in the gut microbiota. ${ }^{30}$

Butyric acid rich fermentations are generally observed with some types of starch that escape digestion in the small intestine. ${ }^{31}$ A recent study conducted by Dan et al. ${ }^{32}$ showed that in vivo colonic fermentation of unripe banana starch resulted in high butyrate production when compared to the control diet, showing also a good correlation between in vitro and in vivo fermentation. Butyrate, in particular, has been targeted as a beneficial SCFA due to its role in the maintenance of colonic homeostasis and anti-inflammatory properties. ${ }^{33-34}$

Propionate fermentation has been reported with diets containing soluble substrates. ${ }^{35}$

Overall, results indicated that AMG-treated starch favored the simultaneous production of relatively larger quantities of propionic and butyric acids compared with the other starches. During starch modification, AMG was greatly active breaking the degree of association between 
glucose molecules. Likewise, considering that AMG hydrolyses $\alpha, 1-4$ bond approximately 500 times faster than the $\alpha, 1-6$ linkage, ${ }^{36}$ it would be right to suppose that the resulting AMG-treated starches would have a highly branched structure and very accessible to fermentation due to its perforated structure. ${ }^{6}$ Its saccharifying activity resulted in more accessible structure for starch digestion and more apparent starch available for fermentation. ${ }^{37-38}$ Furthermore, AM and CGTase were not as active as expected in hydrolyzing glucose linkages, leading less perforated starch granules. These structural features could explain the higher production of SCFA due to higher fermentable starch when modified with AMG.

The fermentation plots of BCFA released were significantly different for non-gelatinized and gelatinized samples (Figure 4), as indicated the statistical analysis (Table 2). The release of BCFA was rather low during the initial 12 hours of fermentation. Only at longer fermentation time a significant amount of these fatty acids were detected, being higher when samples were previously gelatinized (Figure 4). Regarding the effect of enzymes, AMG treated and native starch, in their non-gelatinized state, showed higher amounts of BCFA (Figure 4A). For gelatinized samples (Figure 4B) at $24 \mathrm{~h}$ of fermentation, only a significantly high value was found for the AMG treated starch. Again, results indicated higher metabolic activity in the AMG-treated starches, which agrees with their highest hydrolyzed structure previously mentioned. Moreover, its likely high branched structure would lead higher amount of BCFA. In contrast to SCFA, BCFA have been associated with undesirable protein fermentation, leading to many metabolites that are potentially toxic to the host and associated with carcinogenic effects in the colon. ${ }^{39}$ However, in batch in vitro fermentation systems such as that employed in this study, high BCFA production can also result from higher total metabolic activity of the microbiota. ${ }^{22}$ Because AMG also resulted in high butyrate production, which arises from 
carbohydrate fermentation and not protein fermentation, it appeared that the high BCFA arising from the AMG-treated starch was due to higher metabolic activity and not due to lack of carbohydrate substrate.

Hence, modifications induced by enzymatic treatment of starch granules had a significant effect on in vitro digestion and fermentation pattern. The presence of short chain accessible saccharides besides the starch granules significantly affected the digestion stage, reducing starch digestion. CGTase treated starch was the most hydrolyzed by digestive enzymes in the presence of the sugars released by the enzyme. Conversely, only the structural shape of treated starches affected the fecal fermentation stage, thus the susceptibility of the gut microbiota concerning the balance between SCFA and BCFA production. AMG enzymatically modified starch resulted in enhanced total SCFA production and greater polysaccharide utilization by human fecal microbiota. These results indicates that modification by enzymes of the native starch was accomplished and the possibility to obtain changes in the starch structure leading to a higher butyrate and propionate production improving gut health. Future studies should determine the utilization impact of enzymatically modified samples by AMG as an ingredient in food formulations.

\section{ABBREVIATIONS}

AM-NW: unwashed enzymatically modified sample with $\alpha$-amylase

AM-W: washed enzymatically modified sample with $\alpha$-amylase

AMG-NW: unwashed enzymatically modified sample with amyloglucosidase

AMG-W: washed enzymatically modified sample with amyloglucosidase

BCFA: Branch chain fatty acid 
267 CGT-NW: unwashed enzymatically modified sample with cyclodextrin glycosyltransferase 268 CGT-W: washed enzymatically modified sample with cyclodextrin glycosyltransferase

269 G: gelatinized

270 N: Native corn starch

271 NG: non-gelatinized

272 SCFA: Short chain fatty acid

273 ACKNOWLEDGEMENT

274 A. Dura would like to thank predoctoral fellowship from Spanish Ministry of Economy and 275 Competitiveness. This research was performed in part at the Department of Food Science \& 276 Technology, University of Nebraska-Lincoln, Lincoln, NE, USA.

277 Funding

278 Authors acknowledge the financial support of the Spanish Ministry of Economy and 279 Competitiveness (AGL2014-52928-C2-1-R) and the European Regional Development Fund 280 (FEDER). 


\section{REFERENCES}

1. Rosell, C.M; Dura, A. Enzymes in food and beverage processing. In Baking Industries; Chandrasekaran M., Ed; CRC Press. Taylor \& Francis Group, 2015; pp171-195.

2. Zhang, B.; Cuia, D.; Liua, M.; Gonga, H.; Huanga, Y.; Hana, F. Corn porous starch: Preparation, characterization and adsorption property. Int. J. Biol. Macromol. 2012, 50, 250-256. 3. Dura, A.; Rosell, C.M. Enzymes in baking. In Microbial enzyme technology in food applications. Ray RC \& Rosell CM., Eds;. CRC Press. Taylor \& Francis Group, 2016; pp295314.

4. van der Maarel, M. J. E. C.; van der Veen, B.; Uitdehaag, J. C. M.; Leemhuis, H.; Dijkhuizen, L. Properties and applications of starch-converting enzymes of the $\alpha$-amylase family. $J$. Biotechnol. 2002, 94, 137-155.

5. Dura, A.; Blaszczak, W.; Rosell, C. M. Functionality of porous starch obtained by amylase or amyloglucosidase treatments. Carbohydr. Polym. 2014, 101, 837-845.

6. Benavent-Gil, Y.; Rosell, C.M. Comparison of porous starches obtained from different enzyme types and levels. Carbohydr. Polym. 2017, 117, 533-540.

7. Dura, A.; Rosell, C. M. Physico-chemical properties of corn starch modified with cyclodextrin glycosyltransferase. Int. J. Biol. Macromol. 2016, 87, 466-472.

8. Gérard, C.; Colonna, P.; Buleón, A.; Planchot V. Amylolysis of maize mutant starches. J. Sci. Food Agri. 2001, 81, 1281-1287.

9. Uthumporna, U.; Zaidulb, I.S.M.; Karima, A.A. Hydrolysis of granular starch at sub gelatinization temperature using a mixture of amylolytic enzymes. Food Bioprod. Proc. 2010, 88, 47-54. 
10. Ao, Z.; Simsek, S.; Zhang, G.; Chalam, M. V.; Reuhs, B.L.; Hamaker, B. R. Starch with a slow digestion property produced by altering its chain length, branch density, and crystalline structure. J. Agric. Food Chem. 2007, 55, 4540-4547.

11. Zhang, H.; Tiana, Y.; Bai, Y.; Xu, X.; Jin, Z. Structure and properties of maize starch processed with a combination of $\alpha$-amylase and pullulanase. Int. J. Biol. Macromol. 2013, 52, $38-44$.

12. Brahma, S.; Martinez, I.; Walter, J.; Clarke, J.; Gonzalez, T.; Menon, R.; Rose, D.J. Impact of dietary pattern of the fecal donor on in vitro fermentation properties of whole grains and brans. J. Functional Foods 2017, 29, 281-289.

13. Yang, J.; Rose, D.J. The impact of long-term dietary pattern of fecal donor on in vitro fecal fermentation properties of inulin. Food \& Function 2016, 7,1805-1813.

14. Yang, J.; Rose, D.J. Long term dietary pattern of fecal donor correlates with butyrate production and markers of protein fermentation during in vitro fecal fermentation. Nutr. Res. 2014. $34,749-759$.

15. Tolhurst, G.; Heffron, H.; Lam, Y. S. Sh011-chain fatly acids stimulate glucagon-like peptide-1 secretion via the G-proteincoupled receptor FFAR2. Diabetes. 2012, 61, 364-371.

16. Maslowski, K. M.; Vieira, A. T.; Ng, A.; Kranich, J.; Sierro, F.; Yu, D.; Schilter, H.C.; Rolph, M. S.; Mackay, F.; Artis, D.; Xavier, R. J.; Teixeira, M. M.; Mackay, C. R. Regulation of inflammatory responses by gut microbiota and chemoattractant receptor GPR43. Nature. 2009, $461,1282-1286$.

17. Louis, P.; Hold, G.L.; Flint, H.J. The gut microbiota, bacterial metabolites and colorectal cancer. Nature Rev. Microbiol. 2014, 12, 661-672. 
18. Flint, H.J.; Scott, K.P.; Louis, P.; Duncan, S.H. The role of the gut microbiota in nutrition and health. Nature Rev. Gastro. Hepat. 2012, 9, 577-589.

19. Bernalier-Donadille, A. Fermentative metabolism by the human gut microbiota. Gastr. Clin. Biol. 2010, 34, 16-22.

20. Adam, A.; Lopez, H.W.; Tressol, J-C.; Leuillet, M.; Demignea, C.; Reämeäsy, C. Impact of whole wheat flour and its milling fractions on the fecal fermentations and the plasma and liver lipids in rats. J. Agric. Food Chem. 2002, 50, 6557-6562.

21. Hughes, S. A.; Shewry, P. R.; Li, L.; Gibson, G. R.; Sanz, M. L.; Rastall, R. A. In vitro fermentation by human fecal microflora of wheat arabinoxylans. J. Agric. Food Chem. 2007, 55, 4589-4595.

22. Rose, J. D.; Patterson, J. A.; Hamaker, B. R. Structural differences among alkali-soluble arabinoxylans from maize (Zea mays), rice (Oryza sativa), and wheat (Triticum aestivum) brans influence human fecal fermentation profiles. J. Agric. Food Chem. 2010, 58, 493-499.

23. Arcila, J.A.; Weier, S.A.; Rose, J.V. Changes in dietary fiber fractions and gut microbial fermentation properties of wheat bran after extrusion and bread making. Food Res. Int. 2015, 74, 217-223.

24. Thompson, L.U.; Maningat, C.C.; Woo, K.; Seib, P.A. In vitro digestion of rs4-type resistant wheat and potato starches, and fermentation of indigestible fractions. Cereal Chem. 2011, 88, 7279.

25. Yang, J.; Keshavarzian, A.; Rose, D. J. Impact of dietary fiber fermentation from cereal grains on metabolite production by the fecal microbiota from normal weight and obese individuals. J Med Food. 2013, 16, 862-867. 
26. Arcila, J. A.; Rose, D. J. Repeated cooking and freezing of whole wheat flour increases resistant starch with beneficial impacts on in vitro fecal fermentation properties. J. Funct. Foods. 2015, 12, 230-236.

27. Kimura, A; Robyt, J. F. Reaction of enzymes with starch granules: Enhanced reaction of glucoamylase with gelatinized starch granules. Carbohyd. Res. 1996, 288, 233-240. 28. Sonnenburg, E.D.; Sonnenburg, J.L. Starving our microbial self: the deleterious consequences of a diet deficient in microbiota-accessible carbohydrates. Cell Metab. 2014, 20, 779-786.

29. David, L.A.; Maurice, C.F.; Carmody, R.N.; Gootenberg, D.B.; Button, J.E.; Wolfe, B.E.; Ling, A.V.; Devlin, A.S.; Varma, Y.; Fischbach, M.A.; Biddinger, S.B.; Dutton, R.J.; Turnbaugh, P.J. Diet rapidly and reproducibly alters the human gut microbiome. Nature. 2014, $505,559-563$.

30. Duncan, S.H.; Belenguer, A.; Holtrop, G.; Johnstone, A.M.; Flint, H.J.; Lobley, G.E. Reduced dietary intake of carbohydrates by obese subjects results in decreased concentrations of butyrate and butyrate-producing bacteria in feces. Appl. Environ. Microbiol. 2006, 73, 10731078.

31. Bernalier, A.; Dore, J.; Durand, M. Biochemistry of fermentations. In Colonic Microbiota, Nutrition and Health; Gibson, G. R., Roberfroid, M. B., Eds.; Kluwer Academic Publishers: Dordrecht, The Netherlands, 1999; pp 37-53.

32. Dan, M.C.T.; Cardenette, G.H.L.; Sardá, F. A. H.; Giuntini, E.B.; Bello-Pérez, L.A.; Carpinelli, A.R.; Lajolo, F.M.; Menezes, E.W. Colonic fermentation of unavailable carbohydrates from unripe banana and its influence over glycemic control. Plant Foods Hum. Nutr. 2015, 70, 297-303. 
33. Zhou, J.; Hegsted, M.; McCutcheon, K. L.; Keenan, M. J.; Xi, X.; Raggio, A. M.; Martin, R.J. Peptide YY and proglucagon mRNA expression patterns and regulation in the gut. Obesity. 2006, 14, 683-689.

34. Soliman, M. M.; Ahmed, M. M.; Salah-Eldin, A. E.; Abdel-Aal, A.A. Butyrate regulates leptin expression through different signaling pathways in adipocytes. J. Vet. Sci. 2011, 12, 319323.

35. Levrat, M. A.; Moundras, C.; Younes, H.; Morand, C.; Demigné, C.; Rémésy, C.

Effectiveness of resistant starch, compared to guar gum, in depressing plasma cholesterol and enhancing fecal steroid excretion. Lipids. 1996, 31, 1069-75.

36. Davies, G.J.; Wilson, K.S.; Henrissat, B. Nomenclature for sugar-binding subsites in glycosyl hydrolases. Biochem J. 1997. 321, 557-559.

37. Kimura, A.; Robyt, J. F. Reaction of enzymes with starch granules: kinetics and products of the reaction with glucoamylase. Carbohydr. Res. 1995, 277, 87-107.

38. Brewer, L. R.; Cai, L.; Shi, Y. C. Mechanism and enzymatic contribution to in vitro test method of digestion for maize starches differing in amylose content. J. Agric. Food Chem. 2012, $60,4379-4387$.

39. Thorburn, A. N.; Macia, L.; Mackay, C. R. Diet, metabolites, and "western-lifestyle" inflammatory diseases. Immunity. 2014, 40, 833-842. 


\section{Figure captions}

$390 \quad$ Fig 1 Flow diagram for in vitro digestion and fermentation of starch samples.

391 Fig 2 Digested starch content of enzymatically modified samples with (W) or without hydrolysis 392 products (NW). For non-gelatinized (A) and gelatinized (B) samples. N (native), CGTase 393 (Cyclodextrin glycosyltransferase), AM ( $\alpha$-amylase) and AMG (amyloglucosidase). (*) Denotes 394 significant differences $(P<0.05)$.

395 Fig 3 Total short chain fatty acids and individual SCFA (butyrate, propionate and acetate)

396 produced during fermentation of enzymatically modified samples that had been subjected to in 397 vitro digestion. Values pointed with $(*)$ denote significantly different levels $(P<0.05)$. Native

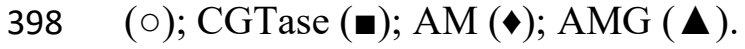

$399 \quad$ Fig 4 Total branch chain fatty acids (BCFA) produced during fermentation of enzymatically modified samples without hydrolysis products, that had been subjected to in vitro digestion previously not gelatinized (A) or gelatinized (B). Values pointed with $(*)$ denote significantly

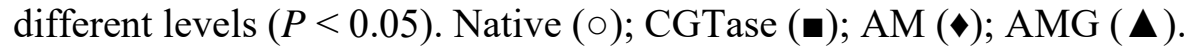


Table 1. Effects Interaction of Type of Enzyme, Washed/Unwashed and Gelatinization in Fecal Fermentation Metabolites and Total Starch after Fecal Fermentation ${ }^{a}$.

\begin{tabular}{lcccccc}
\multicolumn{1}{c}{ Effect $^{\text {a }}$} & BCFA & Acetate & Propionate & Butyrate & SCFA & Starch \\
\hline Washed & 8.21 & 0.83 & 3.37 & 3.11 & 0.31 & $130^{* *}$ \\
Gelatinized & $19.6^{*}$ & 0.26 & 12.0 & 0.11 & 0.29 & $119^{* *}$ \\
Washed*Gelatinized & 4.26 & 2.39 & 0.52 & 0.00 & 0.70 & 13.3 \\
Enzyme & $33.2^{* * *}$ & 2.31 & $27.7 * * *$ & 1.64 & 4.26 & $203^{* * *}$ \\
Washed*Enzyme & 4.80 & 0.07 & 0.53 & 3.09 & 1.91 & $47.8^{* *}$ \\
Gelatinized*Enzyme & $19.04^{* *}$ & 3.50 & 4.65 & 0.79 & 1.94 & $8.32^{*}$ \\
Washed*Gelatinized*Enzyme & 1.81 & 0.14 & 3.06 & 0.03 & 0.04 & $10.6^{*}$ \\
Time & $20.72 * * *$ & $296^{* * *}$ & $141^{* * *}$ & $29.1 * * *$ & $193 * * *$ & $329^{* * *}$ \\
Washed*Time & 1.91 & 0.13 & 1.61 & 2.83 & 1.86 & 2.44 \\
Gelatinized*Time & $6.83^{*}$ & 1.84 & 1.74 & 0.17 & 0.49 & $11^{* *}$ \\
Washed*Gelatinized*Time & 0.89 & 1.57 & 0.54 & 0.10 & 0.84 & 1.19 \\
Enzyme*Time & $12.08^{* * *}$ & 1.37 & $5.16^{* * *}$ & 2.01 & $2.19^{*}$ & $3.35^{* *}$ \\
Washed*Enzyme*Time & 1.50 & 1.45 & $3.53^{*}$ & $3.05^{*}$ & $3.96^{* *}$ & $3^{*}$ \\
Gelatinized*Enzyme*Time & $6.22^{* * *}$ & 0.47 & 0.62 & 0.48 & 0.46 & $2.28^{*}$ \\
Washed*Gelatinized*Enzyme*Time & 0.90 & 0.60 & 1.09 & 0.02 & 0.21 & 0.83 \\
\hline & & & & & & \\
\hline
\end{tabular}

\footnotetext{
${ }^{a}$ Values followed by $P<0.05(*) P<0.01(* *) P<0.001(* *)$
} 
Figure 1

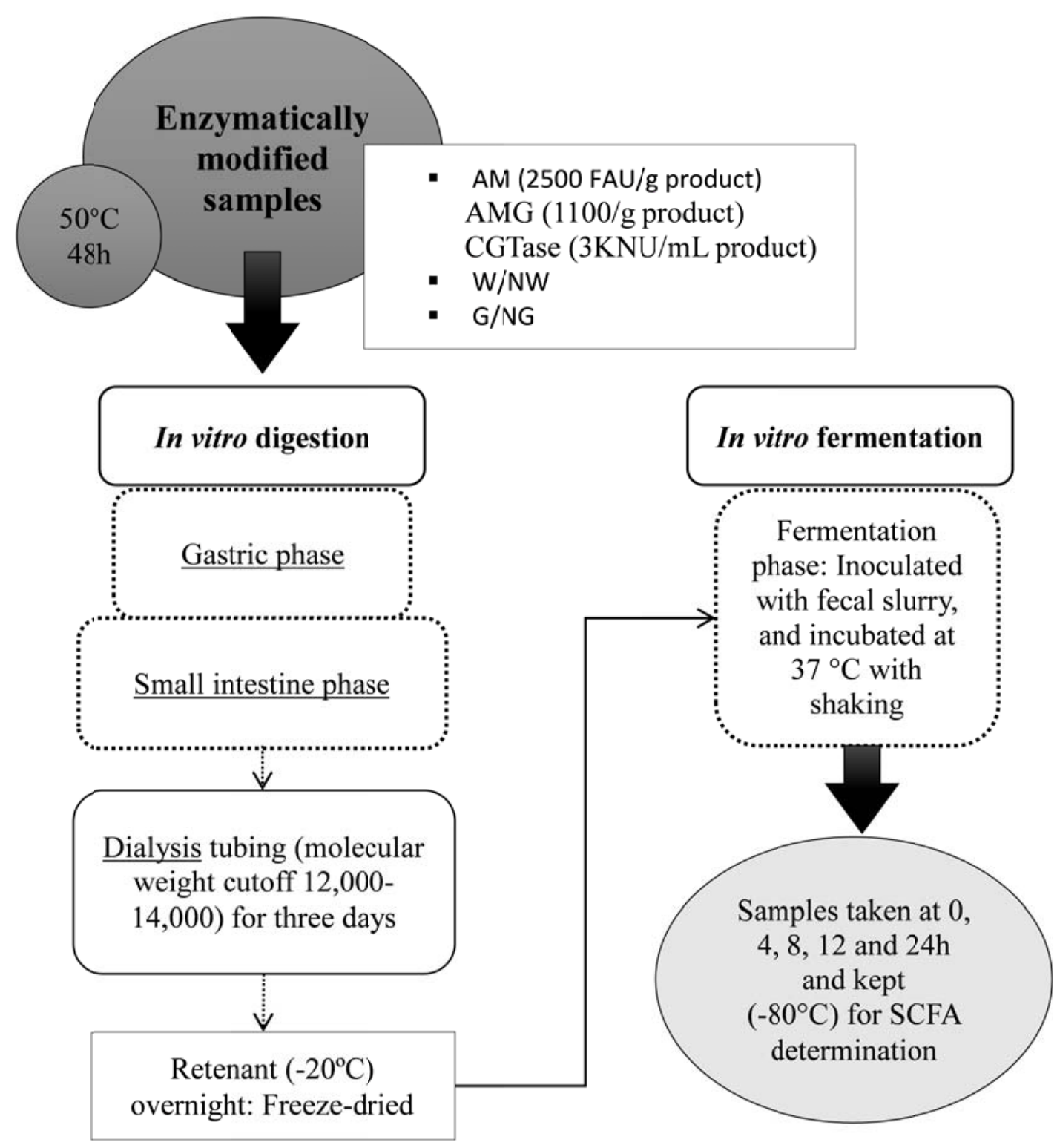


Figure 2

A.

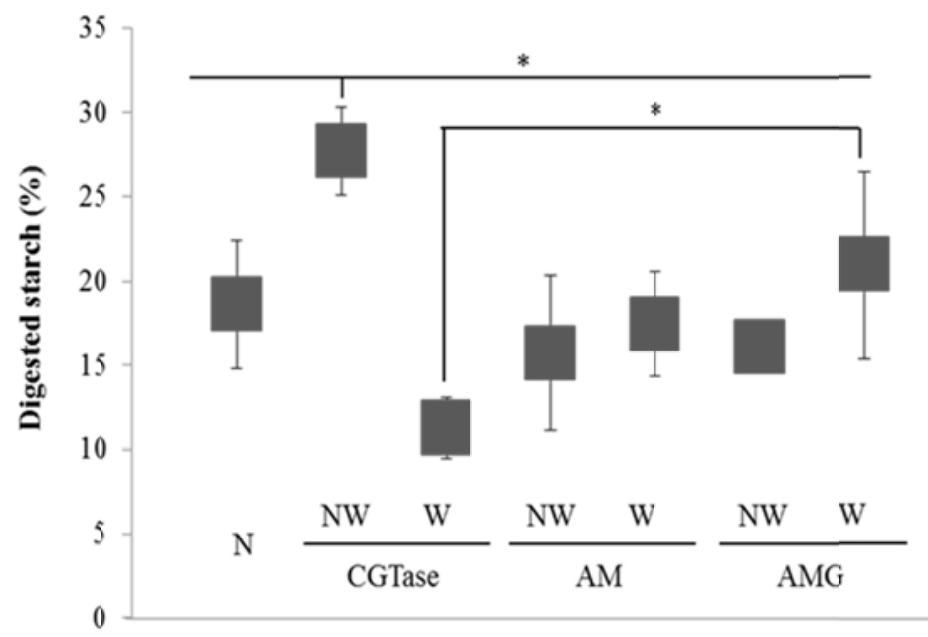

B.

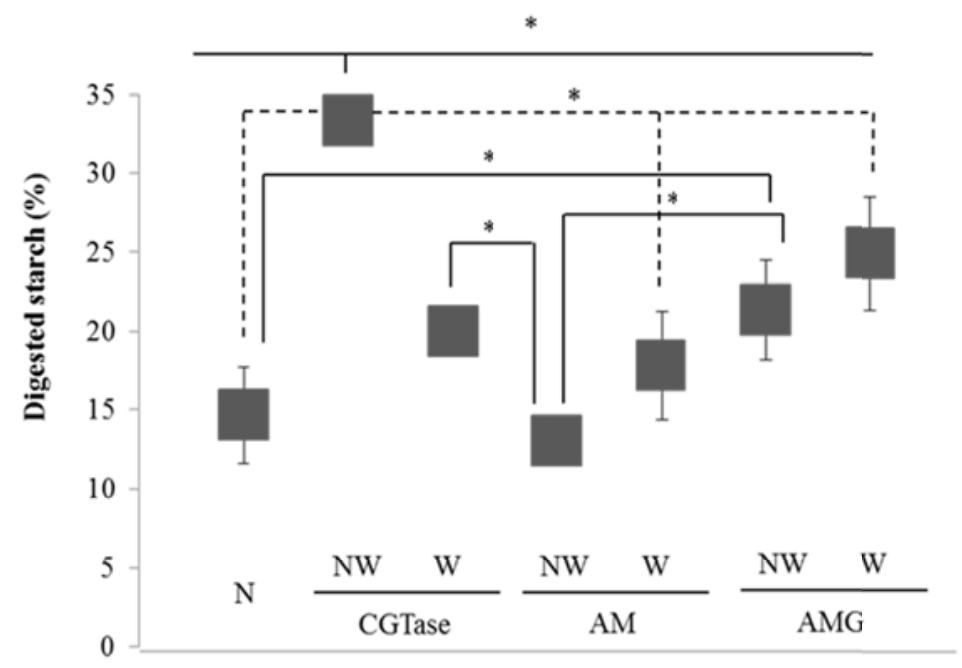


Figure 3
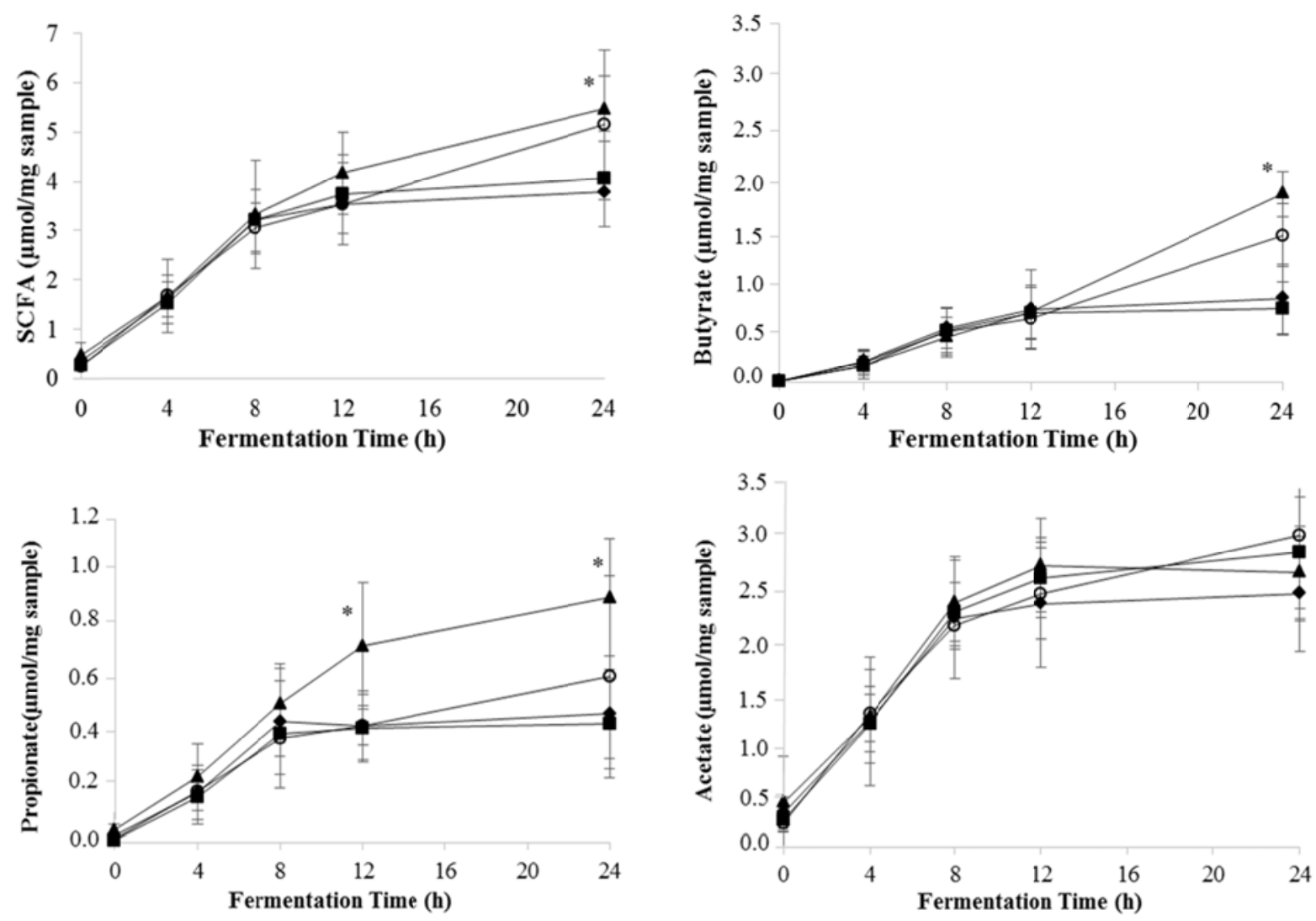
Figure 4

A.

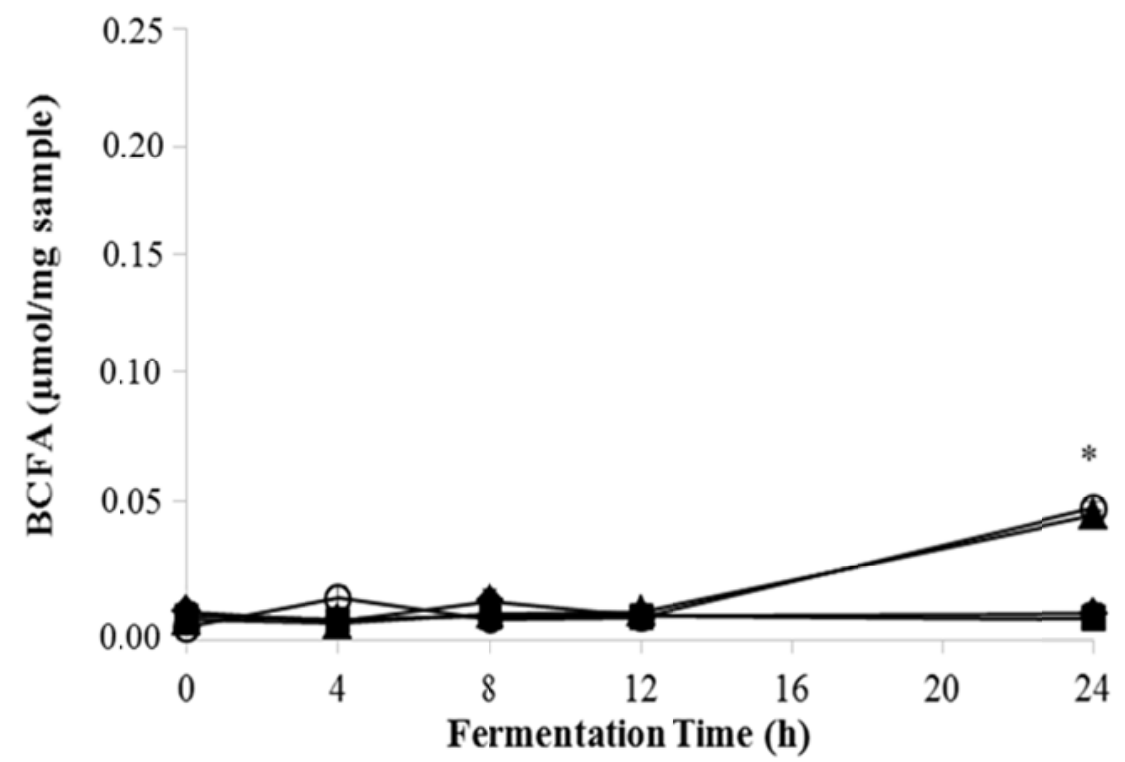

B.

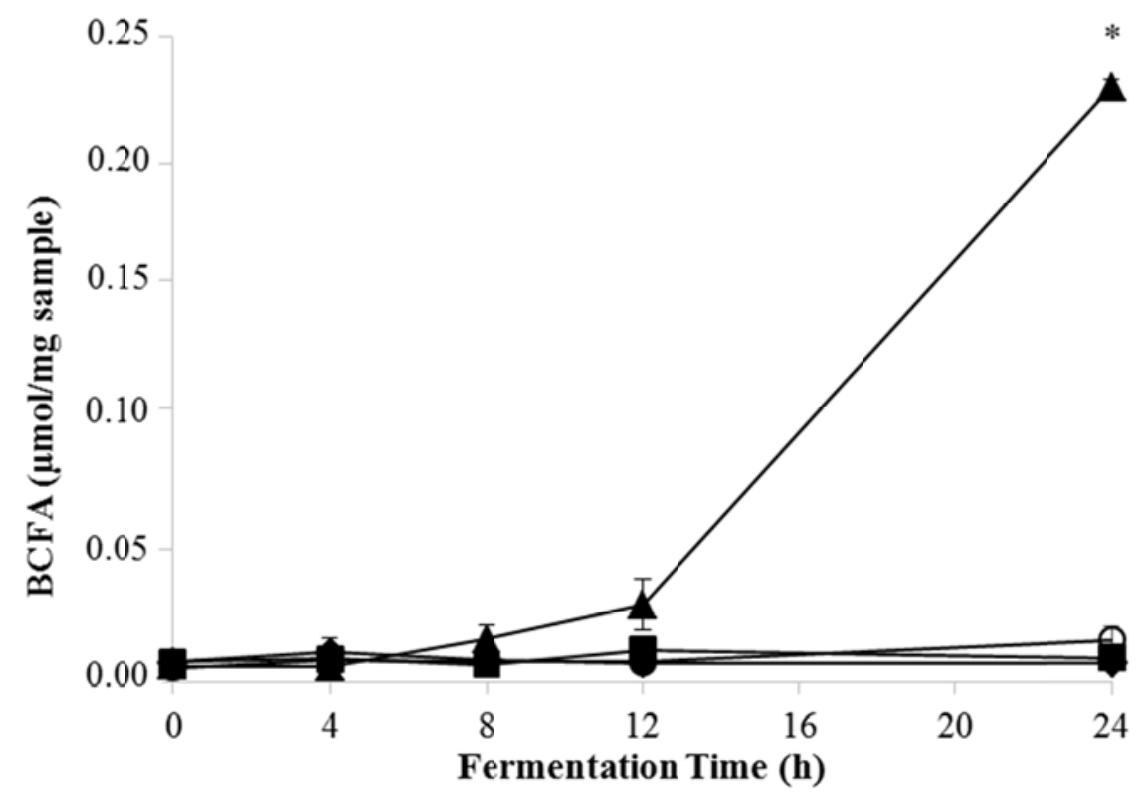


TOC Graphic

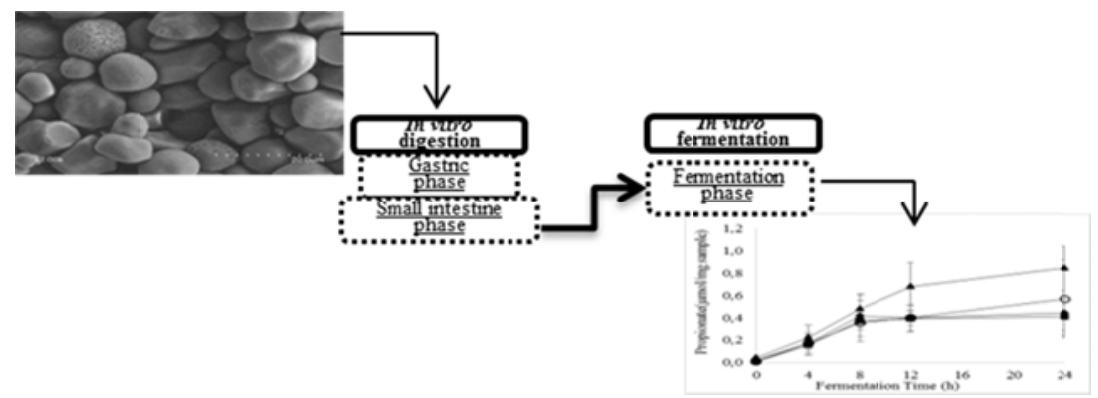

